\title{
The discovery of how gender influences age immunological mechanisms in health and disease, and the identification of ageing gender-specific biomarkers, could lead to specifically tailored treatment and ultimately improve therapeutic success rates
}

\author{
Anna Maria Berghella*, Ida Contasta, Tiziana Del Beato and Patrizia Pellegrini
}

\begin{abstract}
The control of human health and diseases in the elderly population is becoming a challenge, since mean age and life expectation are progressively increasing as well as chronic degenerative diseases. These disorders are of complex diagnosis and they are difficult to be treated, but it is hoped that the predictive medicine will lead to more specific and effective treatment by using specific markers to identify persons with high risk of developing disease, before the clinical manifestation. Peripheral blood targets and biomarkers are currently the most practical, non-invasive means of disease diagnosing, predicting prognosis and therapeutic response. Human longevity is directly correlated with the optimal functioning of the immune system. Recent findings indicate that the sexual dimorphism of T helper (Th) cytokine pathways and the regulation of Th cell network homeostasis are normally present in the immune response and undergoes to adverse changes with ageing. Furthermore, immune senescence affects both men and women, but it does not affect them equally. Therefore, we hypothesize that the comprehension of the interferences between these gender specific pathways, the ageing immunological mechanism in pathological or healthy state and the current therapies, could lead to specifically tailored treatment and eventually improve the therapeutic success rates. Reaching this aim requires the identification of ageing gender-specific biomarkers that could easily reveal the above mentioned correlations.
\end{abstract}

\section{Background}

The progressive increase of mean age and life expectation are correlated to a rise of chronic degenerative diseases such as cancer, cardiovascular, autoimmune or neurodegenerative diseases among the elderly population and these changes will challenge our ability to manage human health and diseases of this category. To this aim, researchers are conducting programs to better understand human ageing and ageing-related diseases and

\footnotetext{
* Correspondence: annamaria.berghella@cnr.it

Consiglio Nazionale delle Ricerche (CNR), Istituto di Farmacologia

Traslazionale (IFT) via G Carducci, 32 - Rotilio Center, 67100 L'Aquila, Italy
}

dysfunction. Chronic degenerative diseases are of complex diagnosis, they are difficult to be treated and they absorb an increasing proportion in the health care budgets worldwide, a phenomenon that will bring social, political, economic and biomedical challenges to future generations [1]. However, recent developments in modern medicine, especially in genetics, proteomics, and informatics, are leading to the discovery of biomarkers that can be used as indicator of disease's risk in healthy subjects.

Predictive medicine uses markers to identify persons with high risk of developing disease before the clinical manifestation. It is hoped that this approach will lead to more specific and effective treatment in the not too 
distant future but this success depends upon the identification of specific biomarkers that can be measured easily and early, from disease onset. Peripheral blood targets and biomarkers are currently the most practical, noninvasive means of disease diagnosing, predicting prognosis and therapeutic response [2].

The identification of ageing gender-specific pathways and biomarkers in peripheral blood would therefore open up an interesting field for research in human health and disease, since gender is related to disease susceptibility [3] and these pathways suffer adverse changes with ageing. Researchers have been shown that sex steroids, for example, influence the regulation of Th cell network balance, shifting the balance toward a Th1 and/ or Th2 type response and both clinical and experimental data have demonstrated the presence of a natural sexual dimorphism in the immune response [3-6]. During their reproductive years, females have a more vigorous cellular and humoral immune response than males and they also have a greater ability to reject tumors and homografts [7-12]. Furthermore, immunosenescence affects both men and women, but it does not affect them equally. Men (all ages) and postmenopausal women exhibit diminished T cell immunity compared to premenopausal women [13]. The decrease in androgens in men with ageing may contribute to their immunosenescence; however, the loss of $\mathrm{T}$ cell function in men with ageing is significantly less dramatic than what has been observed in women [14-16]. There are multiple forms of estrogen: estrone (E1), estradiol (E2) and estriol (E3) are the primary circulating forms. Estradiol binds both estrogen receptor-(ER) $\alpha$ and ER $\beta$ with high and equal affinities, while estrone preferentially binds ER $\alpha$ at a 5-fold higher affinity than ER $\beta$ [17]. However, both pathways are involved in mediating estrogen effects, but ER $\alpha$ and ER $\beta$ exhibit distinct functions within immune cells [18]. In premenopausal women ovary-derived estradiol is the principal circulating estrogen, while estrone is the most abundant circulating estrogen in postmenopausal women and men. In men, testosterone is the primary substrate for estrogen production by peripheral aromatization of androgens precursors, but exhibits a small age-related decrease. Furthermore, most studies failed to observe any significant influence of age on total E2 levels in men [16].

\section{Presentation of the hypothesis}

Therefore, the comprehension of how and why immune responsiveness changes in humans with ageing is essential for developing strategies to prevent or restore deregulated immunity and assure healthy longevity. Here, we advance the hypothesis that the discover of how gender influences age immunological mechanisms in health and disease, and the identification of ageing gender-specific biomarkers, could lead to specifically tailored treatment and ultimately improve therapeutic success rates. We discuss published data on genderdependent immune responses in health and disease states.

It is well established that the gender of a host can significantly affect susceptibility to infection [19]. Epidemiological clinical data and animal models of various human illnesses including sepsis [20] and listeriosis $[21,22]$ reveal that males and females handle infections differently and gender-dependent immune pathways or molecules cause different disease susceptibility in men and women (Table 1).

Women have a more powerful immune system than men: the production of estrogen by females could have a beneficial effect on the innate inflammatory response against bacterial pathogens. Inflammatory caspases, for example, are important effectors of innate immunity [23] and the activity of caspase- 1 is regulated by related inflammatory caspases, namely caspases- 5 and -11 , which activate select inflammasomes [24,25], and caspase-12, which represses caspase- 1 catalysis [26]. Caspase- 12 is expressed in all mammals tested to date, but has acquired deleterious mutation in humans [26]. A single-nucleotide polymorphism introduces a premature stop codon in caspase- 12 in the majority of the population. In a study, a fully humanized mouse that expresses the human caspase-12 rare variant (Csp-12 L) in a mouse casp-12-/- background, was generated and the modalities by which human caspase-12 confers susceptibility to infection [27] was examined. In this study, an unsuspected hormonal regulatory mechanism that governs human Csp-12 L expression during infection, has been identified. These results indicate that through estrogen production, females have a built-in mechanism that prevents Csp-12 L from being expressed, favoring more robust inflammatory and immune responses to pathogens [27].

Sex steroids influence the regulation of Th cell network balance, shifting the balance toward a Th1 and/or Th2 type response. In healthy men and women, the polarization of immune response into Th1 or Th2 cytokines or cellular types is not absolute and the ratio of these cells varies according to physiological demand and clinical conditions [28]. In vivo and in vitro studies in mice have demonstrated that cytokines play a crucial role in maintaining balance in the differentiation of $\mathrm{T}$ cells into Th1 or Th2 types [29]. It was found that pathological conditions arise from abnormalities in the balance between the production of Th1 and Th2 cytokines [30]. It seems that the relative proportion of each cell type depends on the environmental cytokines present during activation [31].

The sex hormones may affect the Th1/Th2 balance, in men and women. However, if we consider the susceptibility difference to autoimmune diseases and infectious diseases 
Table 1 Gender-dependent immune pathways or molecules that causes different disease susceptibility in men and women

\begin{tabular}{lll}
\hline Gender dependent immune pathways or molecules & Disease susceptibility & References \\
\hline Caspase-12 long variant & infectious diseases & Yeretssian G, Proc Natl Acad Sci, 2009, 106:9016 \\
Glucocorticoid receptor pathways & infectious diseases & Wynne O, Stress, 2011, 14:247 \\
Sulfur-dependent detoxification pathways & autism & Al-Yafee YA, BMC Neurol, 2011, 11:139 \\
MyD88-dependent pathway & type 1 diabetes & Sheng H J, Immunol, 2011, 187:1591 \\
STAT5-dependent pathway & human growth disorders & Davey HW, Am J Hum Genet, 1999, 65:959 \\
Toll-like receptor $\mathbf{4}$ & chronic pain & Sorge RE, J Neurosci, 2011, 31:15450 \\
B7-H1-dependent pathway & melanoma & Lin PY, J Immunol, 2010, 185:2747 \\
PARP-1-dependent pathway & lupus nephritis & Caricchio R, NIH RePORTER, 2012, 08 01 \\
HLA-DRB1*0401 and HLA-DQ8 & arthritis & Behrens M, J Autoimmun, 2011, 37:95 \\
Rac1, NOX, ROS & kaposi's sarcoma & Goldschmidt CPJ, NIH RePORTER, 2010, 05 01 \\
CTLA4 & hepatitis C & Schott E, J Hepatol. 2007, 46:372 \\
Toll-like receptor 7 & chronic HCV-infection & Schott E, J Hepatol, 2007, 47:203 \\
Aim2 and p202 proteins & systemic lupus erythematosus & Panchanathan R, Mol Immunol, 2011, 49:273 \\
Retinoid $\mathbf{x}$ receptor alpha & hepatocellular carcinogenesis & Guo M, BMC Genomics, 2008, 9:403 \\
MAPK signaling & systemic lupus erythematosus & Xie H, Arthritis Rheum; 2011, 63:2425 \\
\hline
\end{tabular}

between men and women, the change in the profile of cytokines could be the result of the adaptive response, rather than the cause of the different susceptibility. This implies that hormone-dependent mechanisms operate in the selection of cell phenotypes that regulate the adaptive response and which, if altered, may affect the balance of the Th cells. Induction of Treg cells by estrogen physiological level was, for example found [32]. These authors conclude that estrogen is a potential physiological regulatory factor for the peripheral development of CD4+CD25+ Treg cells [32]: estrogen receptor exist on the CD4+CD25- T cells and the conversion of CD4+CD25- T cells into CD4+CD25+ Treg cells is stimulated by estrogen. Additionally, it was discovered that hormones peripherally activated pro-hormones and regulated the Th1/Th2 balance [33].

There is evidence that sex hormones can affect the immune system and that female and male hormones act in opposing ways [34,35]. For example, Th1 and Th2 responses appear affected by androgenic and estrogenic preponderance, respectively: androgens favor the development of a Th1 response and activation of CD8 cells [36], while estrogens seem to direct the immune system towards Th2 dominance, where B lymphocytes are activated and antibody production flourishes [35]. Pregnancy, a high estrogen state, is of course characterized by Th2 preponderance, and a failure in the establishment of the Th2 dominance has been associated with increased risk for pregnancy loss $[37,38]$.

Gender accounts for important differences in the incidence and prevalence of a variety of age-related diseases. Research finding [39-41] points out that gender is a major variable in the genetics of longevity, suggesting that men and women follow different strategies to reach longevity. These results [41] confirm the age-related remodelling of cytokine network and that variations in pro- or anti-inflammatory cytokines might influence successful ageing and longevity, suggesting that the multiplex analysis of cytokine levels might be useful in defining a successful ageing profile $[41,42]$.

A recent study [43] focusing on gender-dependent immune responses indicates, for the first time, that gender-specific Th cytokine pathways may well be responsible for the different gender-dependent responses to disease and therapy and open up an exciting new field for research. This study supports the concept that a sexual dimorphism of Th cytokine pathways and regulation of Th cell network homeostasis are normally present in the immune response. Antigen presenting cells (APCs) regulate Th cell differentiation and Th cell network homeostasis under resting and activated conditions of the immune system in both men and women, however this effect appears to be exerted through male and female gender-specific and gender-common health pathways (Figure 1).

IFNy and IL6 production pathways are the respectively male and female gender-specific health pathways for the immune response homeostasis and they are targets and/or biomarkers for the passage from health to adenoma and, eventually, to colorectal cancer. IL10 pathway is the common-gender pathway which restores immune system resting homeostasis in both men and women, but only if it is controlled by the above 


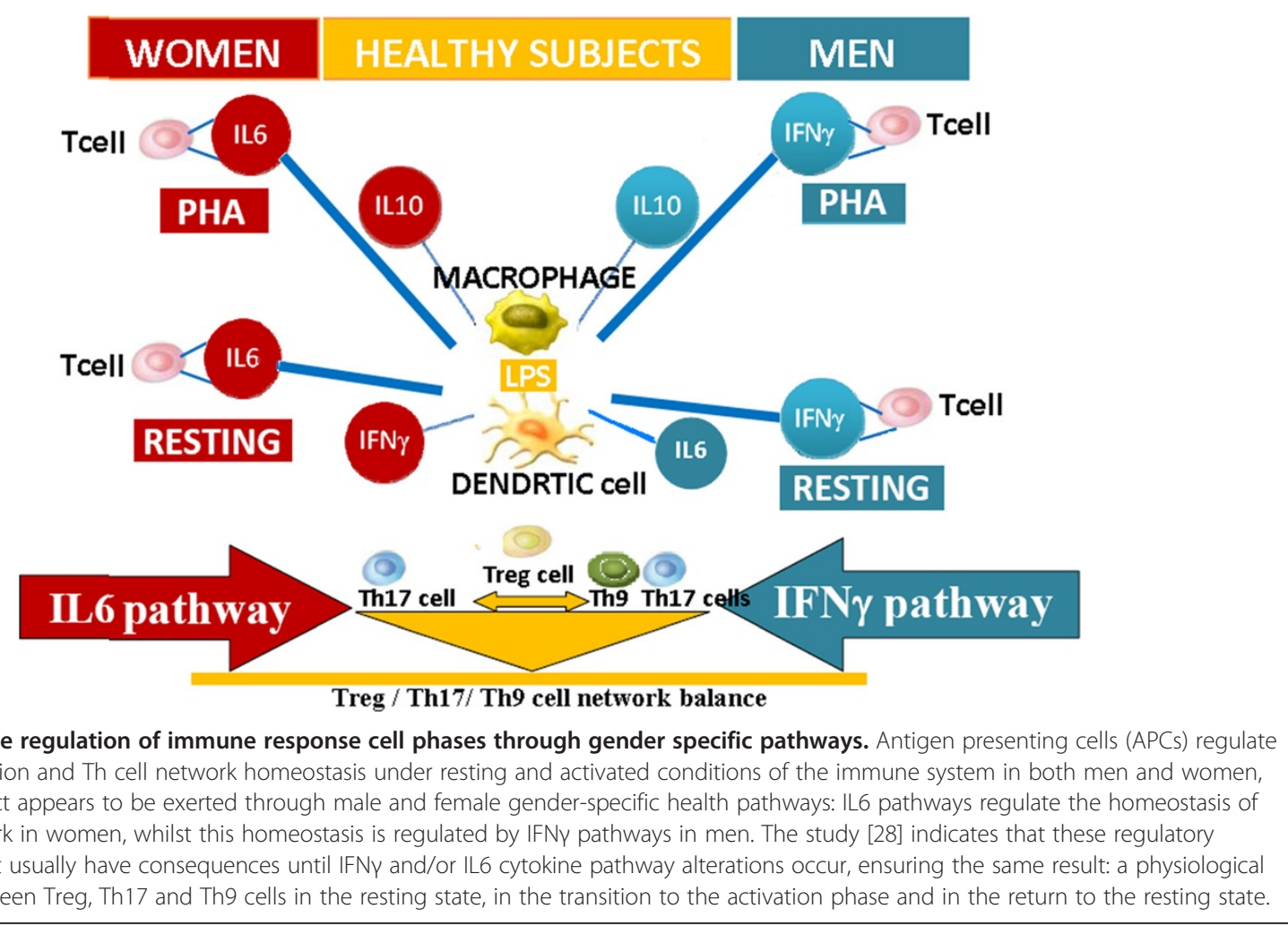

mentioned gender specific pathways; otherwise IL10 pathway is a target/biomarker for cancer progression.

These findings showed that: i) the immune cell production of IL6 in women and IFNY in men decreases with the increasing of the age (Figure 2); ii) age could also be a significant independent factor for IFN $\gamma$ and IL10 in men; iii) whilst in women age appears to be significant for IFN $\gamma$ and the soluble interleukin 6 receptor (sIL6R) molecule, which forms a ligand-receptor complex with IL6, that is capable of stimulating a variety of cellular responses including proliferation, differentiation and activation of inflammatory processes [44].

Figure 2 shows that IFNY level decreases with aging in healthy men, however the IL2 level seems to be a better candidate than IFNY level. To verify the IFNY role as a gender-specific pathway, the authors use their mathematical modeling by excluding IL2 and IFNy cytokines alternately. The graphs obtained from the principal component analysis (Figure 3), clearly define the homeostatic role of IFNy and the IFNy-dependence of IL2. Indeed, excluding IL2 the relationships of the cytokine network are not affected, however the exclusion of IFNy completely reshapes these relationships.

Gender specific variations in cytokine network relationships between pro- or anti-inflammatory cytokines influence the success of the immune response [41]. The results of the above mentioned study [43] showed that the early evolution of immune response is influenced by the positive inter-regulation between production of IFN $\gamma$-IL10 and IL6-IL4 cytokines in men, and the negative interregulation of IL6-IL10 cytokines in women. Similarly, the late evolution of immune response seems to be influenced by the positive inter-regulation between the production of IFN $\gamma$-IL4 in men and by IL6-IFN $\gamma$ in women. These gender specific cytokine network relationships, between pro- or anti-inflammatory cytokines, vary with the increasing of the age (Figure 4) and they are dual gender specific biomarkers that could well be used to develop more specific approaches.

Overall, these findings underline the need for gender specific drugs that could take into account the different regulation system of the immune response, ensuring the same therapeutic result: the return to a physiological homeostasis thanks to the transition from a pathological activation phase to a physiological resting state. Obviously the regulatory differences do not usually have consequences until IFN $\gamma$ and/or IL6 cytokine pathway alterations occur, in which case the consequences for men and women, in terms of pathological mechanisms and disease development, are different. The malfunctioning of gender specific pathways not only compromises the homeostasis of the immune response, but may also cause a pathological polarization of $\mathrm{T}$ cell subsets specific to each sex. In fact, IFN $\gamma$ supports the 


\section{Healthy men: IFN $\gamma \downarrow$ with ageing}

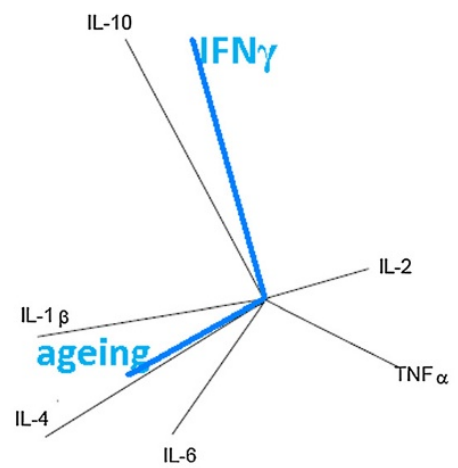

\section{Healthy women: IL6 $\downarrow$ with ageing}

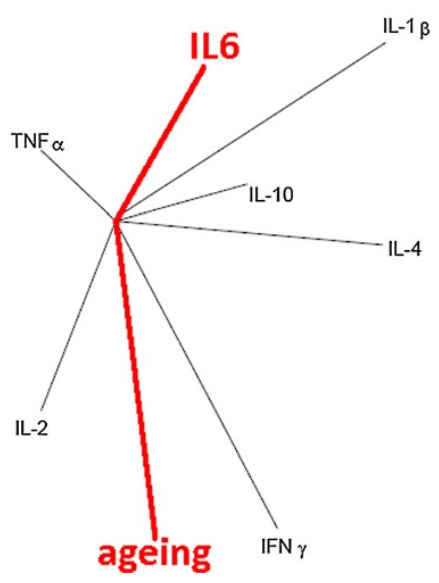

Figure 2 The serum levels of IL6 in women and IFNY in men decrease with increasing age. A group of 66 healthy subjects of Italian people, 33 men and 33 women (blood donors, laboratory staff and relative), were studied [28]. None of the subjects was receiving concurrent drug treatment including widely-used pharmaceuticals, such as salicylates and sex hormones (contraceptive pill, hormone replacement therapy). Distribution of age in male and female groups was the same (mean $\pm \mathrm{SD}=$ $41 \pm 12$ years, compared to mean $\pm S D=41 \pm 15$ years, $p=0.14$ ). In physiological systems components operate as a network and each component varies and co-varies dynamically with respect to one another. Therefore, the identification of physiological pathways, and correlated biomarkers can only be achieved through evaluations that take these fluctuations into account. Using the principal component analysis these authors plotted the network of vectors obtained by analyzing the data matrix of correlation/covariance coefficients of serum cytokines. In these plots, the angle between vectors is inversely proportional to the degree of correlation between vectors; the same vector direction indicates a positive correlation/covariance, the opposite vector direction indicates a negative correlation/covariance. This allows a visualization of the situation under study and is an excellent method for capturing significance from systems biology evaluations. The plots show that the serum levels of IL6 in women and IFNY in men decrease with increasing age. The results of multiple regression analysis confirm that age could also be a significant independent factor for IFNY $(p=0.01)$ and IL10 $(p=0.03)$ in men; whilst in women age appears to be significant for sIL6R $(p=0.002)$ and IFNY $(p=0.04)$. development of Th1 functions [45] promoting cell mediated immunity, while IL6 cytokine supports Th2 responses where B lymphocytes are activated and antibody production flourishes. Additionally, research in this field has shown that it is not a single cytokine that determines a particular response but rather the interaction of individual cytokines within a network.

The results of the above mentioned study [43] indicate that a different gender susceptibility and clinical course in diseases is caused by different Treg, Th17 and Th9 cell polarization (Figure 5) determined by the TGF $\beta$, IL6, IFNy, IL10 and IL4 cytokine pathway interactions which vary between men and women [43]. These findings are, indeed, backed up by the results of other researches indicating that there is a reciprocal development relationship between Treg, Th17 and Th9 cells because: i) TGF $\beta$ triggers the expression of Foxp3 transcription factor in naïve $\mathrm{T}$ cells, generating Treg cells, but ii) IL6 inhibits the TGF $\beta$ driven expression of Foxp3, and TGF $\beta$ together with IL6 induce ROR-gt transcription factor, triggering the developmental program of Th17 cells [46], while ii) IL4 also inhibits TGF $\beta$ induction of Foxp3 expression, but TGF $\beta$ together with IL4 induce the differentiation of Th9 cells, which produce IL9 cytokine. On the other hand, the co-expression of IL9 and IL17 was identified as a novel Th17 function in mediating immune and autoimmune tissue destruction $[47,48]$, also in the central nervous system. Hence, autoimmune disease susceptibility in women, such as multiple sclerosis (MS), could be attributed to the influence of IL6, which plays a key role in autoimmune diseases, since it is a $\mathrm{T}$ cell differentiation switch factor from Tregs to Th17 cells [46,49-51]. The greater likelihood of men developing the primary progressive MS form [52], on the other hand, could be the result of the influence of IFNY on Th9 cell inhibition. In fact, the IL9 receptor complex is constitutively expressed on astrocytes. IL9 induces astrocytes to produce CCL-20 but not other chemokines, including CCL-2, CCL-3, and CXCL-2 [53], suggesting that IL9 induces CCL-20 production by astrocytes to induce the migration of Th17 cells into the CNS. Treg, Th9 and Th17 cells have been shown to be important CD4 $\mathrm{T}$ cell subsets in human autoimmune diseases, including rheumatoid arthritis [54] and MS [55] diseases.

Our recent research on MS disease (article in press) confirms these data, by showing that a sexual dimorphism in autoimmune diseases is the result of different pathways that regulate the Th cell network homeostasis: IL6 pathways in women and IFN $\gamma$ pathways in men. Since women are more susceptible to MS disease and the IL6 has a more significant role in the autoimmune process compared to IFN $\gamma$, it is logical to assume that IL6 pathways are in some way implicated in the prevalence of autoimmune diseases in women. Indeed, our data indicate 


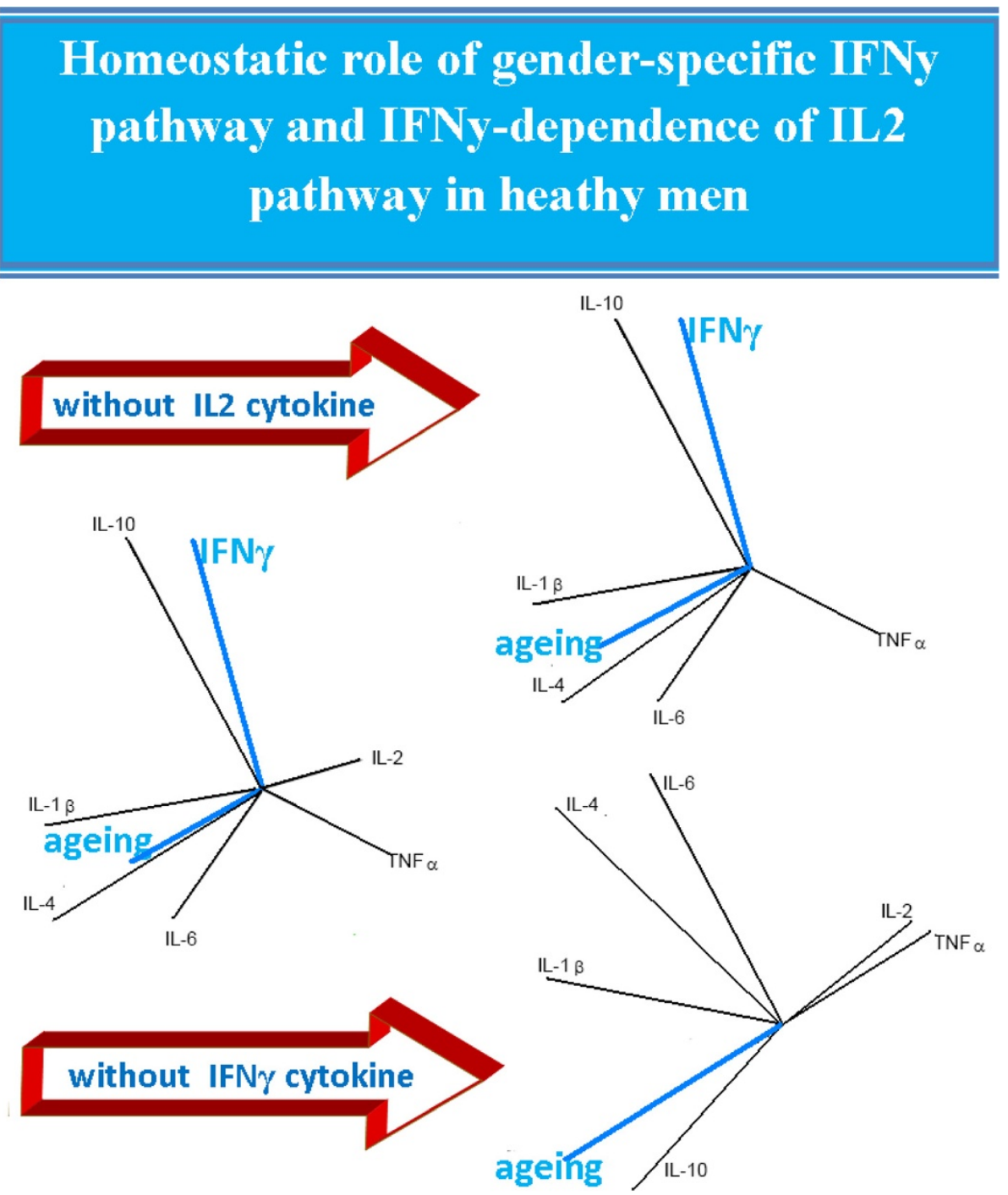

Figure 3 Homeostatic role of IFNy pathway and IFNy-dependence of IL2 pathway in heathy men. In the APC analysis, in heathy men (Figure 2) IFNY level decreases with aging, however the IL2 level seems to be a better candidate than IFNY. The authors use their mathematical modeling to verify the IFNy role as a gender-specific pathway, by excluding from the model IL2 and IFNy alternately. The graphs obtained from this analysis, clearly define the homeostatic role of IFNy and IFNy-dependence of IL2. Indeed, excluding IL2 reports from the network of cytokines are not affected, however the exclusion of IFNy completely reshapes these relationships.

that IL6 pathways are also involved in Treg cell imbalance and an increase in neurological deficit in both men and women groups of MS patients, underlining the autoimmune etiology of MS disease. In further support of differing cytokine pathways in men and women, we noted that the efficacy of IFN $\beta$-treatment in the reestablishment of Th-network balance and delaying the progression of neurological disability is linked to the IL6 pathway in women, but to the IFNY pathway in men.

The specific mechanisms responsible for gender specific disease susceptibility have yet to be clarified. However these data suggest that the answer may lie in the different capacity of cells to defend themselves against oxidative stress [56]. The cells of men and women differ greatly in terms of reactive oxygen species (ROS) production and oxidative stress susceptibility [57] and this appears to be a promising new field of investigation. Oxygen metabolism can lead to the production of ROS in all cell types. All cell types, including lymphocytes and other immune system cells, present antioxidant compounds and enzymes (such as glutathione and thioredoxin reductase) $[58,59]$ to neutralize ROS and to preserve the cell oxidative balance. However, the activities of ROS appear to be regulated differently in males and females and can be directly influenced by sex hormones [57].

In vivo studies have further demonstrated the incapacity in males, but not in females, of maintaining intracellular reduced redox conditions, essential for normal 


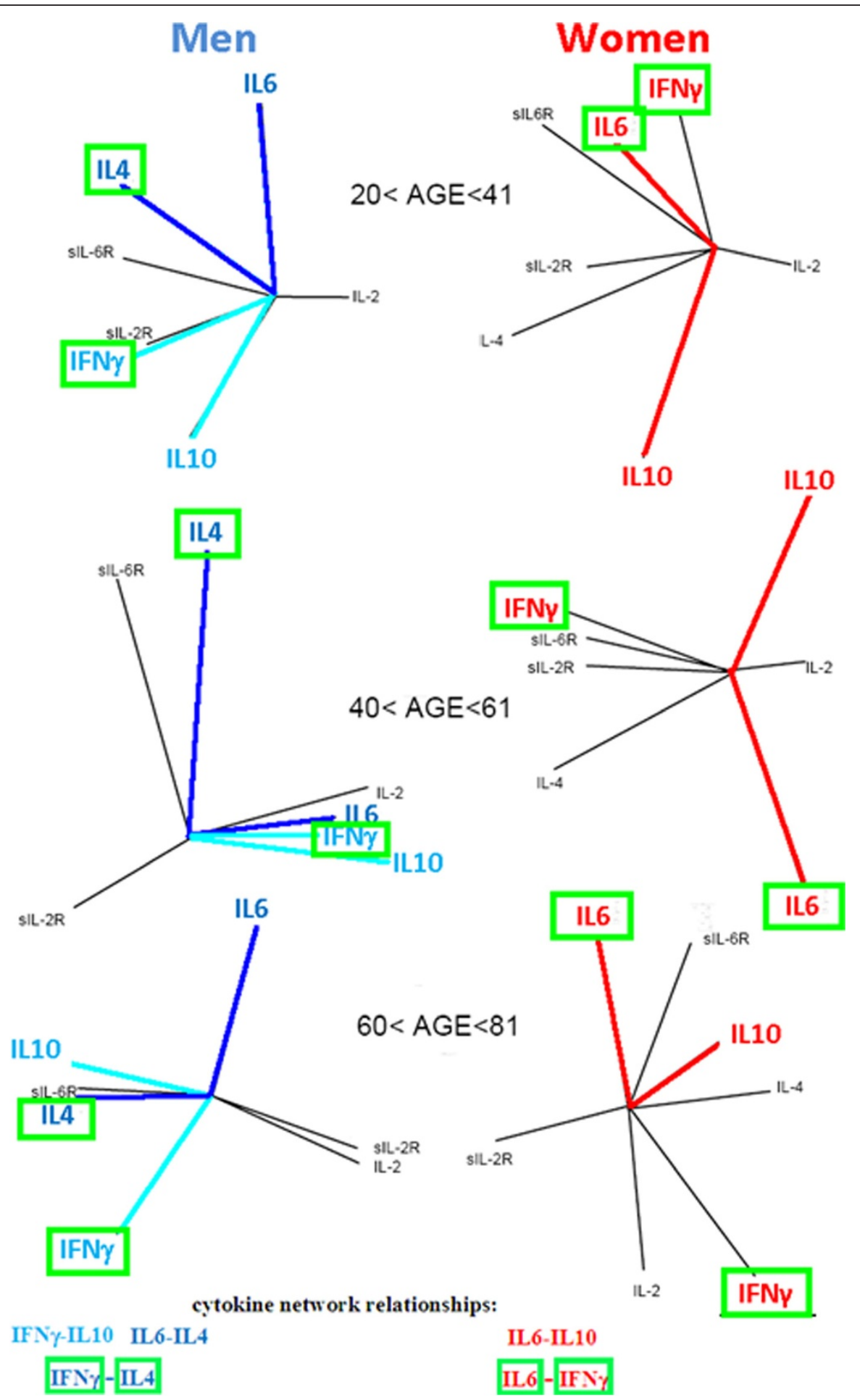

Figure 4 Gender specific ageing variations in cytokine network relationships between pro- or anti-inflammatory cytokines could influence the success of the immune response. The study results indicate that variations in specific cytokine network relationships, between pro- or anti-inflammatory cytokines, regulate immune response homeostasis in healthy state. The early evolution of immune response is controlled by the positive inter-regulation between production of IFNY-IL10 and IL6-IL4 cytokines in men, and the negative inter-regulation of IL6-IL10 cytokines in women. Similarly, the late evolution of immune response seems to be regulated by the positive inter-regulation between the production of IFN $\gamma$-IL4 in men and by IL6-IFN $\gamma$ in women. The principal component analysis has shown that these gender specific cytokine network relationships suffering changes during ageing, which could adversely affect the success of the immune response. Consequently, these cytokine relationships are dual gender specific biomarkers that could well be used to develop more specific approaches in the elderly population. 


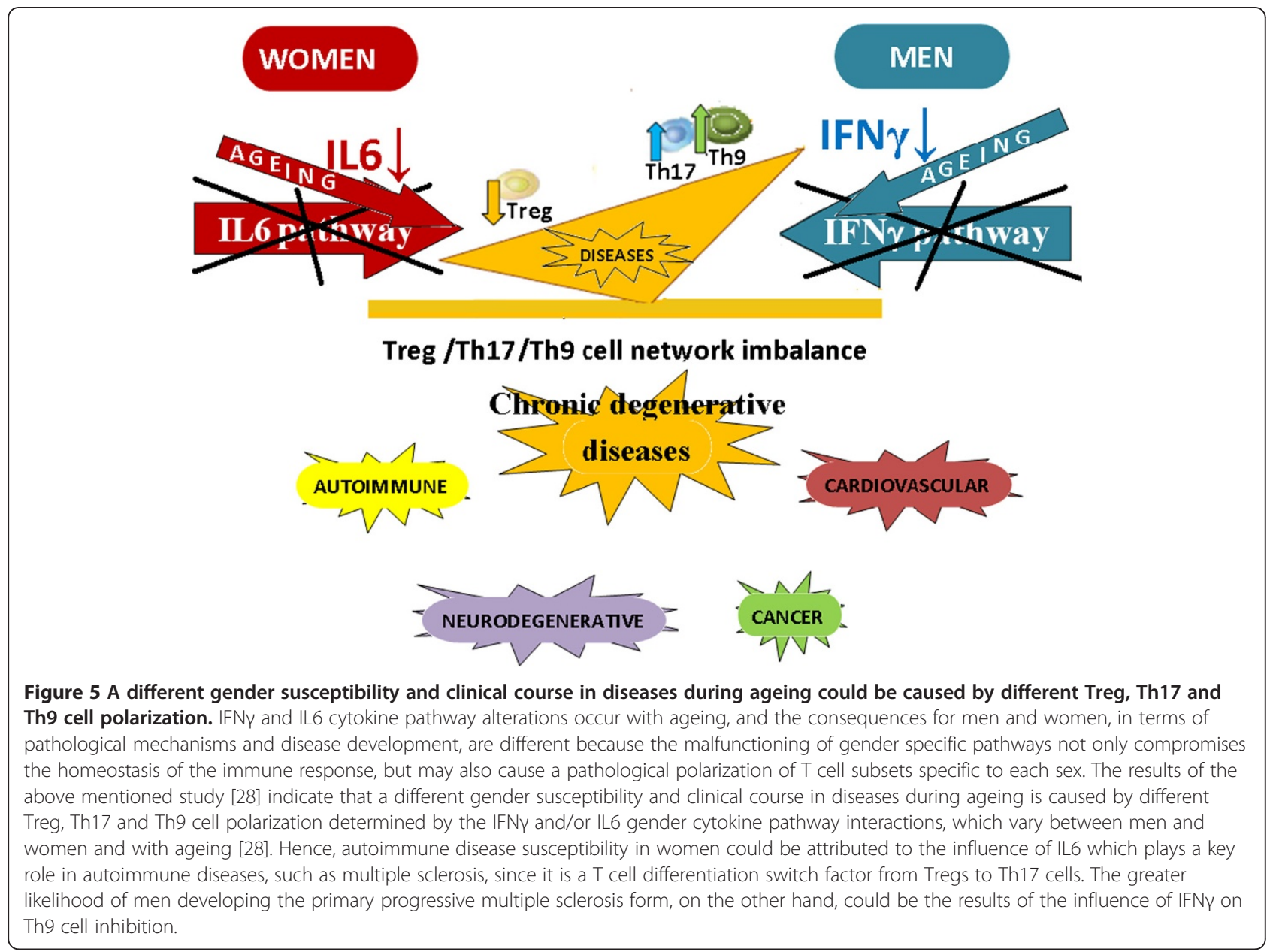

cellular functions [55], and this explains, at least in part, the differences between the two sexes in the maintenance of the immune system homeostasis. In fact, IFNY is a direct stimulator of the gene expression of thioredoxin and thioredoxin reductase (RTrx) system in human $\mathrm{T}$ cells $[59,60]$ and there is a positive feed-back circuit involving IFN $\gamma$ and Trx/RTrx gene expression in the regulation of intracellular reduced oxidative condition, which is essential for the immune response. Subsequently, we can assume that the immunological response through the IFN $\gamma$ pathway in men reduces the intracellular oxidative levels to preserve the cell oxidative balance control. In fact, male cells, as we mentioned, are incapable of maintaining an intracellular reduced oxidative condition.

\section{Implications of the hypothesis}

The comprehension of how and why immune responsiveness changes in humans as they age is essential for developing strategies to prevent or restore deregulated immunity and assure healthy longevity. Research findings showed that:
1. gender is a major variable in the genetics of longevity and men and women follow different strategies to reach longevity [39-41];

2. gender-specific Th cytokine pathways and the regulation of Th cell network homeostasis are normally present in the immune response [43]; IFNY and IL6 production pathways are the respectively male and female gender-specific health pathways for immune response homeostasis; IL10 pathway is the common-gender pathway which restores immune system resting homeostasis in both men and women, but only if it is controlled by the above mentioned gender specific pathways; these regulatory differences do not usually have consequences until IFN $\gamma$ and/or IL6 cytokine pathway alterations occur and IL6 and IFNY pathway suffer adverse changes with ageing $[43,61]$

3. the cytokine network undergoes age-related remodelling [41], variations in pro- or anti-inflammatory cytokines might influence 
successful ageing and longevity and the multiplex analysis of cytokine levels are useful in defining a successful ageing profile [41,42];

4. the early evolution of immune response is influenced by the positive inter-regulation between production of IFN $\gamma$-IL10 and IL6-IL4 cytokines in men, and the negative inter-regulation of IL6-IL10 cytokines in women [43]. Similarly, the late evolution of immune response seems to be influenced by the positive inter-regulation between the production of IFN $\gamma$-IL4 in men and by IL6-IFNY in women. These cytokine relationships are "dual gender specific biomarkers" that could well be used to develop more specific approaches.

These independent findings support a new perspective of research: the comprehension of how the different gender pathways interfere with ageing and lead to diseases, and how they interfere with the success of current therapies, is of utmost importance in translational medicine physiological treatment. The above mentioned gender-specific Th cytokine pathways and the "dual gender specific cytokines" are peripheral blood ageing gender-specific targets and biomarkers and would open up an interesting field for research in human health and disease.

Indeed, important points support the validity of our hypothesis and feasibility of this new research perspective, looking for ageing gender specific biomarkers that can be identified from the general ageing population.

One of the biggest problems in finding good biomarkers of ageing is that so many measures are indicators of disease rather than measures of "normal" function [62]. Some gerontologists believe that ageing is not a process or set of processes, but rather is the cumulative effect of damage that makes the body unable to response to external stimuli, others believe that ageing is itself a disease. Searching for biomarkers of ageing only makes sense if there really are relevant biological processes to be measured [62].

The process of ageing is a complex phenomenon, it is the consequence of the deterioration of more than one system [62,63]. Ageing, in the biological sense, is the loss of the ability to maintain homeostasis, that means the loss of the ability to respond to environmental challenges, such as heat or cold or infection, by a) overcoming the challenge and b) restoring normal function. Loss of homeostatic ability can occur at the level of the whole organism or in one or more of its parts.

This assumption leads to the conviction that a "panel" of biomarkers that reflects the condition of an array of critical systems is needed in order to assess the biological age of any organism. Therefore, a specific "panel" could be useful if the biomarkers are predictive of the deterioration in multiple systems. A "panel" of such biomarkers would allow predictions of health span that might be quite different from lifespan.

A low-grade systemic inflammation characterizes ageing and this pro-inflammatory status underlies biological mechanisms responsible for age-related inflammatory diseases [64]. On the other hand, clinical and epidemiological studies show a strong association between chronic infection, inflammation and age-related disease. A wide range of factors, including smoking, infections, obesity, genetics and declining of sex hormone levels may contribute to systemic low-grade inflammatory activity in older individuals [65]. Progressive increase of mean age and life expectancy parallels, in fact, an increment of chronic inflammation which are an index of risk for chronic degenerative diseases such as cancer, cardiovascular, autoimmune or neurodegenerative diseases among the elderly. The goal must be to identify non-symptomatic individuals with high susceptibility to the disease, which can benefit from protocols for the prevention or early intervention.

Therefore, predictive medicine must 1) as a first step anticipate the deleterious effect of chronic degenerative diseases using markers to identify people with chronicinflammatory status and 2) then use high risk markers to predict the developing of a specific chronic degenerative disease before the clinical manifestation. This innovative approach may offer substantial advantages, since the promise of personalized medicine is to preserve individual health in people with high risk by starting early treatment or prevention protocols [1].

On the basis of the above considerations a feasible plan, predictive and diagnostic for physiological ageing and age linked diseases, can be identified by using as first step a) the immunological "panel" of "dual gender biomarkers" and then b) the high risk markers for developing a specific chronic degenerative disease, generated by molecular, genetic and neuroimaging techniques.

The use of the immunological "panel" of "dual gender biomarkers" as first approach to identify people with pro-inflammatory status is supported by the following reasons:

1. The levels of "dual gender-specific cytokines" are dual-biomarkers of gender-specific Th cytokine pathways. They are measurable "non-disease related aging biomarkers", useful to indicate if the subject conditions comply with an healthy state or with a transition to a chronic-inflammatory state. These biomarkers select the population according to gender that, as we reported, is the main biomarker for biological and functional differences in the immune response, susceptibility to disease and therapeutic response. "Dual gender biomarkers" represent gender specific biomarkers for homeostasis in the immune 
response and the human longevity is directly correlated with the optimal functioning of the immune system.

2. The gender-specific Th cytokine pathways are gender-specific target underlying healthy biological processes, that can be related to relevant functions and to be measured in healthy people.

3. Cytokines are easily measurable biomarkers, as they reside in peripheral blood.

4. These immunological biomarkers are predictive of the deterioration in multiple systems that would allow predictions of health span that might be quite different from lifespan.

With this evaluation system, the normal level of "dual gender specific cytokines" in all "dual gender biomarkers", is an index for an healthy state because it is an indicator of the ability to maintain physiological homeostasis (Figure 5). Level variation of "dual gender specific cytokines" (for example IFN $\gamma$-IL10, IL6-IL and IFN $\gamma$-IL4 in men) in a direction that agrees with the positive or negative sign of their healthy relationship (in this case positive), it is an index for a transient inflammation, because it is still an indicator of immune system homeostasis; the level variation of "dual gender specific cytokines" in a direction that does not agree (in this case negative) with the positive or negative sign of their healthy relationship, it is index for a chronic inflammation, because it is an indicator of loss of homeostatic ability (Figure 6).

These new "panel" of biomarkers, predictive for a) the inflammation state and b) the specific type of disease involved, may lead to reduces diseases incidence rate and to distinguish clinical subtypes of a single disease to better tailor both, potential prevention strategies and/or early intervention protocols for chronic degenerative diseases, maintaining an acceptable quality of life.

Further study will validate or refute this new hypothesis. Operative proposals for the heath care systems are now needed to verify potential benefits in predictive medicine.

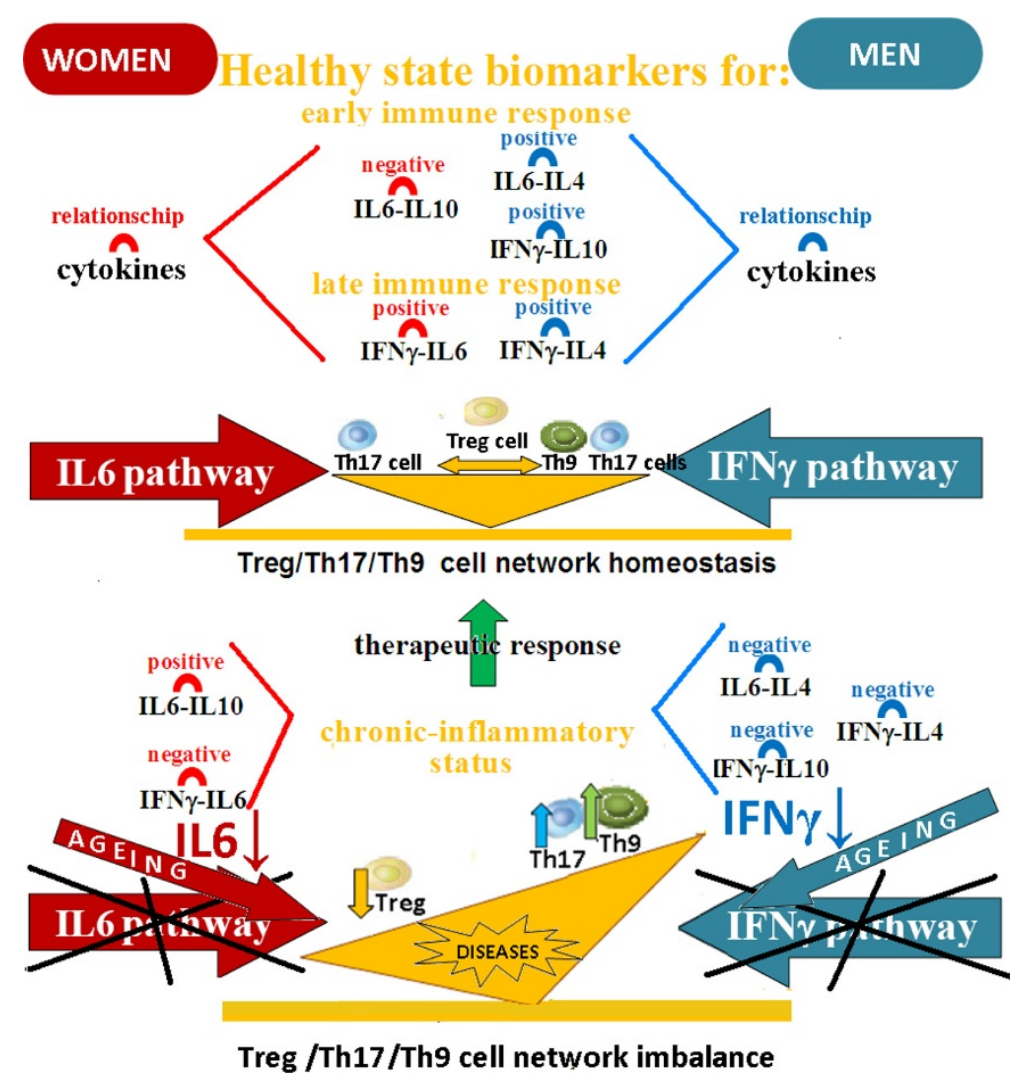

Figure 6 The normal level of "dual gender specific cytokines" in all "dual gender biomarkers", is an index for an healthy state because it is an indicator of the ability to maintain physiological homeostasis. Level variation of "dual gender specific cytokines" (for example IFNYIL10, IL6-IL4 and IFNY-IL4 in men) in a direction that agrees with the positive or negative sign of their healthy relationship (in this case positive), it is an index for a transient inflammation, because it is still an indicator of immune system homeostasis. Level variation of "dual gender specific cytokines" in a direction that does not agree (in this case negative) with the positive or negative sign of their healthy relationship, it is index for a chronic inflammation, because it is an indicator of loss of homeostatic ability. 


\section{Competing interests}

The authors declare that they have no competing interests.

\section{Authors' contributions}

AMB designed the research, analyzed and interpreted data, wrote the manuscript. IC and PP contributed to design the research and analyze data; TDB contributed to analyze data. All authors read and approved the final manuscript.

\section{Acknowledgements}

We would like to thank the Mayor, Dr. Giuseppe Marulli, and the Deputy Mayor, Mr. Virgilio Lerza, and the administrative staff of Capestrano Town Council (L'Aquila-Italy), for giving us office space following the loss of our building in the earthquake in L'Aquila in 2009. We also thank Enzo Secinaro, student of medicine, for his support in the control of the English script of text.

Received: 9 August 2012 Accepted: 28 October 2012

Published: 13 November 2012

\section{References}

1. Licastro F, Caruso C: Predictive diagnostics and personalized medicine for the prevention of chronic degenerative diseases. Immun Ageing 2010, 16:7.

2. Martin KJ, Fournier MV, Reddy GP, Pardee AB: A need for basic research on fluid-based early detection biomarkers. Cancer Research 2010, 70:5203-6.

3. Liu LY, Schaub MA, Sirota M, Butte AJ: Sex differences in disease risk from reported genome-wide association study findings. Hum Genet 2012, 131:353-64

4. Grossman CJ: Possible underlying mechanisms of sexual dimorphism in the immune response, fact and hypothesis. Journal of Steroid Biochemistry 1989, 34:241-251.

5. Schuurs $A H$, Verhuel $H A$ : Effects of gender and sex steroids on the immune response. Journal of Steroid Biochemistry 1990, 35:157-172.

6. Cannon JC, St-Pierre BA: Gender differences in host defense mechanisms. Journal of Psychiatric Research 1997, 31:99-113.

7. Rhodes K, Scott A, Markhan RL, Monk-Jones ME: Immunological sex differences. Annals of Rheumatic Diseases 1969, 28:104-120.

8. Butterworth MB, McClellan B, Alansmith M: Influence of sex on immunoglobulin levels. Nature 1967, 214:1224-1225.

9. Terres G, Morrison SL, Habicht GH: Quantitative difference in the immune response between male and female mice. Proceedings of the Society for Experimental Biology and Medicine 1968, 127:664-667.

10. Morell V: Zeroing in on how hormones affect the immune system. Science 1995, 269:773-775.

11. Homo-Delarche F, Fitzpatrick F, Christeff N, Nunez E, Bach JF, Dardenne M: Sex steroids, glucocorticoids, stress and autoimmunity. The Journal of Steroid Biochemistry and Molecular Biology 1991, 40:619-637.

12. Grossman CJ: Regulation of the immune system by sex steroids. Endocrine Reviews 1984, 5:435-455.

13. Pietschmann P, Gollob E, Brosch S, Hahn P, Kudlacek S, Willheim M, Woloszczuk W, Peterlik M, Tragl KH: The effect of age and gender on cytokine production by human peripheral blood mononuclear cells and markers of bone metabolism. Exp Gerontol 2003, 38:1119-27.

14. Khosla S, Melton L, Atkinson EJ, O'Fallon WM, Klee GG, Riggs BL: Relationship of serum sex steroid levels and bone turnover markers with bone mineral density in men and women: a key role of bioavailable estrogen. J Clin Endocrinol Metab 1998, 83:2266-2274.

15. Vermeulen A, Kaufman JM, Goemaere S, van Pottelberg I: Estradiol in elderly men. Aging Male 2002, 5:98-102

16. Dolomie-Fagour L, Gatta B, Nguyen TDT, Corcuff JB: Bioavailable estradiol in man: Relationship with age and testosterone. Clin Chim Acta 2008, 398:145-147.

17. Zhu BT, Han GZ, Shim JY, Wen Y, Jiang XR: Quantitative structure- activity relationship of various endogenous estrogen metabolites for human estrogen receptor $\alpha$ and $\beta$ subtypes: Insights into the structural determinants favoring a differential subtype binding. Endocrinol 2006, 147:4132-4150

18. Li J, McMurray RW: Effects of estrogen subtype-selective agonists on immune functions in ovariectomized mice. Int Immunopharmacol 2006, 6:1413-1423.
19. Terres G, Morrison SL, Habicht GS: A quantitative difference in the immune response between male and female mice. Proc Soc Exp Biol Med 1968, 127:664-667.

20. Beery TA: Sex differences in infection and sepsis. Crit Care Nurs Clin North Am 2003, 15:55-62.

21. Gellin BG, Broome CV: Listeriosis. JAMA 1989, 261:1313-1320.

22. Pasche B, Kalaydjiev S, Franz TJ, Kremmer E, Gailus-Durner V, Fuchs H: Hrabé de Angelis M, Lengeling A, Busch DH: Sex-dependent susceptibility to Listeria monocytogenes infection is mediated by differential interleukin10 production. Infect Immun 2005, 73:5952-5960.

23. Petrilli V, Dostert C, Muruve DA, Tschopp J: The inflammasome:Adanger sensing complex triggering innate immunity. Curr Opin Immunol 2007 19:615-622.

24. Martinon F, Burns K, Tschopp J: The inflammasome: A molecular platform triggering activation of inflammatory caspases and processing of prolLbeta. Mol Cell 2002, 10:417-426.

25. Wang S, Miura M, Jung YK, Zhu H, Li E, Yuan J: Murine caspase-11, an ICEinteracting protease, is essential for the activation of ICE. Cell 1998, 92:501-509.

26. Saleh M, Mathison JC, Wolinski MK, Bensinger SJ, Fitzgerald P, Droin N, Ulevitch RJ, Green DR, Nicholson DW: Enhanced bacterial clearance and sepsis resistance in caspase-12 deficient mice. Nature 2006, 440:1064-1068.

27. Yeretssian G, Doiron K, Shao W, Leavitt BR, Hayden MR, Nicholson DW, Saleh M: Gender differences in expression of the human caspase-12 long variant determines susceptibility to Listeria monocytogenes infection. Proc Natl Acad Sci U S A 2009, 106:9016-20.

28. Mosmann TR, Schumacher JH, Street NF, Budd R, O'Gara A, Fong TAT, Bond MW, Moore KWM, Sher A, Fiorentino DF: Diversity of cytokine synthesis and function of mouse CD4+ T cells. Immunol Rev 1991, 123:209-29.

29. Coffman RL, Varkila K, Scott $P$, Chatelain R: Role of cytokines in the differentiation of CD4+ T-cell subsets in vivo. Immunol Rev 1991, 123:189-207.

30. Peltz G: A role for CD4+ T cell subsets producing a selective pattern of lymphokines in the pathogenesis of human chronic inflammatory and allergic diseases. Immunol Rev 1991, 123:23-35.

31. Murray JS, Madri J, Pasqualini T, Bottomly K: Functional CD4 T cell subset interplay in an intact immune system. J Immunol 1993, 150:4270-6.

32. Tai $P$, Wang J, Jin $H$, Song $X$, Yan J, Kang Y, Zhao L, An X, Du X, Chen X, Wang S, Xia G, Wang B: Induction of regulatory $T$ cells by physiological level estrogen. J Cell Physiol 2008, 214:456-64.

33. Rook GA, Hernandez-Pando R, Lightman SL: Hormones, peripherally activated prohormones and regulation of the Th1/Th2 balance. Immunol Today 1994, 15:301-3.

34. Whitacre CC: Sex differences in autoimmune disease. Nat Immunol 2001, 9:777-80.

35. McCarthy M: The "gender gap" in autoimmune disease. Lancet 2000, 356:1088.

36. Beageley KW, Gockel CM: Regulation of innate and adaptive immunity by the female sex hormones oestradiol and progesterone. FEMS Immunol Med Microbiol 2003, 38:13-22

37. Gleicher N: Some thoughts on the reproductive autoimmune failure syndrome (RAFS) and $\mathrm{TH}-1$ versus $\mathrm{TH}-2$ immune responses. Am J Reprod Immunol 2002, 48:252-4.

38. Yakoo T, Takakuwa K, Ooki I, Kikuchi A, Tamura M, Tanaka K: Alterations of $\mathrm{TH} 1$ and $\mathrm{TH} 2$ cells by intracellular cytokine detection in patients with unexplained recurrent abortion before and after immunotherapy with the husband's mononuclear cells. Fertil Steril 2006, 85:1452-8.

39. Lio D, Scola L, Crivello A, Colonna-Romano G, Candore G, Bonafè M, Cavallone L, Franceschi C, Caruso C: Gender-specific association between -1082 IL-10 promoter polymorphism and longevity. Genes Immun 2002, 3:30-3.

40. Franceschi C, Motta L, Valensin S, Rapisarda R, Franzone A, Berardelli M, Motta M, Monti D, Bonafè M, Ferrucci L, Deiana L, Pes GM, Carru C, Desole MS, Barbi C, Sartoni G, Gemelli C, Lescai F, Olivieri F, Marchegiani F, Cardell M, Cavallone L, Gueresi P, Cossarizza A, Troiano L, Pini G, Sansoni P, Passeri G, Lisa R, Spazzafumo L, Amadio L, Giunta S, Stecconi R, Morresi R, Viticchi C, Mattace R, De Benedictis G, Baggio G: Do men and women follow different trajectories to reach extreme longevity? Italian Multicenter Study on Centenarians. Aging 2000, 12:77-84.

41. Balistreri CR, Candore G, Accardi G, Bova M, Buffa S, Bulati M, Forte Gl, Listi F, Martorana A, Palmeri M, Pellicano M, Vaccarino L, Scola L, Lio D, Colonna- 
Romano G: Genetics of longevity. Data from the studies on Sicilian centenarians. Immun Ageing 2012, 9:8.

42. Palmeri M, Misiano G, Malaguarnera M, Forte GI, Vaccarino L, Milano S, Scola L, Caruso C, Motta M, Maugeri D, Lio D: Cytokine serum profile in a group of Sicilian nonagenarians. J Immunoassay Immunochem 2012, 33:82-90.

43. Pellegrini P, Contasta I, Del Beato T, Ciccone F, Berghella AM: Genderspecific cytokine pathways, targets, and biomarkers for the switch from health to adenoma and colorectal cancer. Clin Dev Immunol 2011, doi:819724.

44. Jones SA, Horiuchi S, Topley N, Yamamoto N, Fuller GM: The soluble interleukin 6 receptor: mechanisms of production and implications in disease. FASEB J 2001, 15:43-58.

45. Thomson A: The Cytokine Handbook. San Diego, Calif, USA: Academic; 1994

46. Bettelli E, Carrier Y, Gao W, Korn T, Strom TB, Oukka M, Weiner HL, Kuchroo VK: Reciprocal developmental pathways for the generation of pathogenic effector TH17 and regulatory T cells. Nature 2006, 441:235-238.

47. Korn T, Mitsdoerffer M, Croxford AL, Awasthi A, Dardalhon VA, Galileos G, Vollmar P, Stritesky GL, Kaplan MH, Waisman A, Kuchroo VK, Oukka M: IL-6 controls Th17 immunity in vivo by inhibiting the conversion of conventional T cells into Foxp3+ regulatory T cells. Proc Natl Acad Sci U S A 2008, 105:18460-5.

48. Nowak EC, Weaver CT, Turner H, Begum-Haque S, Becher B, Schreiner B, Coyle AJ, Kasper LH, Noelle RJ: IL-9 as a mediator of Th17-driven inflammatory disease. J Exp Med 2009, 206:1653-1660.

49. Oukka M: Th17 cells in immunity and autoimmunity. Annals of the Rheumatic Disease 2008, 67:26-9.

50. Cua DJ, Kastelein RA: TGFß a 'double agent' in the immune pathology war. Nature Immunology 2006, 7:557-9.

51. Korn T, Anderson AC, Bettelli E, Oukka M: The dynamics of effector T cells and Foxp3+ regulatory $T$ cells in the promotion and regulation of autoimmune encephalomyelitis. Journal of Neuroimmunology 2007, 191:51-60.

52. Greer JM, McCombe PA: Role of gender in multiple sclerosis: clinical effects and potential molecular mechanisms. J Neuroimmunol 2011, 234:7-18.

53. Zhou $Y$, Sonobe $Y$, Akahori $T$, Jin $S$, Kawanokuchi J, Noda M, Iwakura $Y$, Mizuno T, Suzumura A: IL-9 promotes Th17 cell migration into the central nervous system via CC chemokine ligand- 20 produced by astrocytes. J Immunol 2011, 186:4415-21.

54. Murphy CA, Langrish CL, Chen Y, Blumenschein W, McClanahan T, Kastelein RA, Sedgwick JD, Cua DJ: Divergent pro- and anti-inflammatory roles for IL-23 and IL-12 in joint autoimmune inflammation. J Exp Med 2003, 198:1951-1957.

55. Tzartos JS, Friese MA, Craner MJ, Palace J, Newcombe J, Esiri MM, Fugger L: Interleukin-17 production in central nervous system infiltrating $\mathrm{T}$ cells and glial cells is associated with active disease in multiple sclerosis. Am J Pathol 2008, 172:146-155.

56. Du L, Bayir H, Lai Y, Zhang X, Kochanek PM, Watkins SC, Graham SH, Clark RS: Innate gender-based proclivity in response to cytotoxicity and programmed cell death pathway. The Journal of Biological Chemistry 2004 279:38563-38570

57. Ortona E, Margutti P, Matarrese P, Franconi F, Malorni W: Redox state, cell death and autoimmune diseases: a gender perspective. Autoimmunity Reviews 2008, 7:579-584.

58. Hansen JM, Go YM, Jones DP: Nuclear and mitochondrial compartmentation of oxidative stress and redox signaling. Annual Review of Pharmacology and Toxicology 2006, 46:215-34.

59. Song JJ, Lee YJ: Differential role of glutaredoxin and thioredoxin in metabolic oxidative stress-induced activation of apoptosis signal regulating kinase 1. Biochemical Journal 2003, 373:845-53.

60. Kim SH, Oh J, Choi JY, Jang JY, Kang MW, Lee CE: Identification of human thioredoxin as a novel IFN-gamma-induced factor: mechanism of induction and its role in cytokine production. BMC Immunology 2008, 9.64 doi:10.1186/1471-2172-9-64.

61. Pellegrini P, Contasta I, Berghella AM, Del Beato T, Casciani CU, Adorno D: The TH1 and TH2 cytokine network in healthy subjects: suggestions for experimental studies to create prognostic and diagnostic indices for biotherapeutic treatments. Cancer Biother Radiopharm 2000, 15:267-78.
62. Sprott RL: Biomarkers of aging and disease: introduction and definitions. Exp Gerontol 2010, 45:2-4.

63. Barker $P E$, Murthy M: Biomarker Validation for Aging: Lessons from mtDNA Heteroplasmy Analyses in Early Cancer Detection. Biomark Insights 2009, 4:165-79.

64. Vasto S, Carruba G, Lio D, Colonna-Romano G, Di Bona D, Candore G, Caruso C: Inflammation, ageing and cancer. Mech Ageing Dev 2009, 130:40-5.

65. Krabbe KS, Pedersen M, Bruunsgaard H: Inflammatory mediators in the elderly. Exp Gerontol 2004, 2004(39):687-699.

doi:10.1186/1742-4933-9-24

Cite this article as: Berghella et al:: The discovery of how gender influences age immunological mechanisms in health and disease, and the identification of ageing gender-specific biomarkers, could lead to specifically tailored treatment and ultimately improve therapeutic success rates. Immunity \& Ageing 2012 9:24.

\section{Submit your next manuscript to BioMed Central and take full advantage of:}

- Convenient online submission

- Thorough peer review

- No space constraints or color figure charges

- Immediate publication on acceptance

- Inclusion in PubMed, CAS, Scopus and Google Scholar

- Research which is freely available for redistribution 